\title{
Jean-François Bert (dir.), Henri Hubert et la sociologie des religions
}

Liège, Presses universitaires de Liège, coll. «Religions », 2015, 330 p.

Pierre Lassave

\section{(2) OpenEdition}

\section{Journals}

Édition électronique

URL : http://journals.openedition.org/assr/28186

DOI : $10.4000 /$ assr.28186

ISSN : $1777-5825$

Éditeur

Éditions de l'EHESS

Édition imprimée

Date de publication : 31 décembre 2016

Pagination : 270

ISSN : 0335-5985

Référence électronique

Pierre Lassave, « Jean-François Bert (dir.), Henri Hubert et la sociologie des religions », Archives de sciences sociales des religions [En ligne], 176 | octobre-décembre 2016, mis en ligne le 17 juillet 2017, consulté le 24 septembre 2020. URL : http://journals.openedition.org/assr/28186 ; DOI : https:// doi.org/10.4000/assr.28186

Ce document a été généré automatiquement le 24 septembre 2020.

(C) Archives de sciences sociales des religions 


\title{
Jean-François Bert (dir.), Henri Hubert et la sociologie des religions
}

Liège, Presses universitaires de Liège, coll. «Religions », 2015, 330 p.

\author{
Pierre Lassave
}

\section{RÉFÉRENCE}

Jean-François Bert (dir.), Henri Hubert et la sociologie des religions, Liège, Presses universitaires de Liège, coll. « Religions », 2015, 330 p.

1 Après l'hécatombe de la Première Guerre, qui a durement touché l'école durkheimienne, Marcel Mauss n'a eu de cesse de publier les travaux de ses collègues disparus. Tel fut le cas des Mélanges de sociologie religieuse et de folklore de Robert Hertz en 1928 puis des travaux d'histoire culturelle sur les Celtes et les Germains d'Henri Hubert, son ami proche, prématurément décédé à l'âge de cinquante-cinq ans en 1927 (sur leur gémellité intellectuelle, voir notre recension d'un précédent essai, Archives de Sciences Sociales des Religions, $\left.\mathrm{n}^{\circ} 160,2012\right)$. Mutatis mutandis, le recueil de morceaux choisis ici par Jean-François Bert et ses collaborateurs (Christine Lorre, Rafael Benthien, Nicolas Meylan) prolonge le geste en rééditant des écrits d'Hubert enfouis dans des dictionnaires, manuels et préfaces qui datent de plus d'un siècle déjà. Textes sur la magie, le sacré, le temps et le culte des héros qui n'ont pas pris beaucoup de rides et méritaient d'être rassemblés sous le thème de la sociologie des religions au sens large. On connaît surtout Hubert à travers ses articles coécrits avec Mauss, lignes désormais classiques sur les processus sacrificiels, la pratique magique et la théorie anthropologique du sacré. Leur exégèse éclairée par les correspondances internes au "club Totem-Tabou " (Durkheim-Hubert-Mauss) nous a déjà rendus sensibles aux nuances de conception entre ses trois membres éminents. Hubert et Mauss avaient ainsi insisté sur l'ambivalence du sacré qui, bien qu'en principe séparé du profane, ne communique pas moins avec lui par le biais, par exemple, des pratiques sacrificielles; ils avaient également pris quelque distance avec l'assimilation par Durkheim du mana, 
principe de force, au sacré, considérant ce dernier comme une espèce dont le mana serait seulement le genre.

La présente réédition prolonge ce paysage de nuances sémantiques. Hubert insiste ainsi sur l'interdit qui qualifie le sacré et par extension toute religion comme administration du sacré par interdictions rituelles. C'est un des messages forts qui transparait de son introduction à la traduction française du Manuel d'histoire des religions de P.-D. Chantepie de la Saussaye (1904), universitaire hollandais alors très influent dans les académies européennes. Message qui rejoint la première définition de Durkheim (1898) partant du caractère obligatoire ou coercitif de toute religion. Mais cette introduction est riche d'enseignements sous divers aspects. Hubert se garde ainsi de toute définition prématurée du mot religion dont le sens varie éminemment d'un temps et d'un lieu à l'autre. Il se montre également réservé à l'égard des théories des écoles anglaises ou allemandes qui s'appuient uniquement sur l'inférence spéculative d'un rapport humain à l'indicible ou d'un sentiment de lien avec quelque esprit ou puissance surplombants. Mieux vaut d'abord considérer les innombrables faits et gestes qui spécifient ce qui se dit religieux, soit le règlement des relations entre les hommes et entre ceux-ci et les êtres considérés comme sacrés ou frappés d'interdits, «le tout formant une vaste société et les dernières sortes de relation étant sociales au même titre que les premières. " D'où la nécessité de décrire par le menu ces relations d'un continent et d'une époque à l'autre pour étayer les comparaisons. « Sage éclectisme » qui ne doit pas moins s'arrêter un temps pour fixer les concepts qui relancent l'étude, telle cette tendance de l'auteur à voir plutôt dans la religion l'administration du tabou et dans la magie l'art de faire avec le mana. Travail sur le fil entre faits et sens qui nous vaut de belles lignes vaguement surannées et prudemment métaphoriques qui méritent le détour : «C'est au cours de la vie sociale que la religion a poussé. Elle a fleuri en prières, sacrifices, mythologie, morale et métaphysique, sans oublier les pousses folles de la magie. L'arbre est d'une seule venue, mais ses maîtresses branches sont puissantes ; les plus lourdes, courbées jusqu'à terre, y ont pris racine, comme celle d'un banian, et leur ramure cache le tronc. Suivant les saisons et les points de vue, la figure de cet arbre varie à tel point qu'on a souvent peine à le reconnaître; les philosophes et les historiens, qui s'y sont trompés, l'ont pris pour une forêt d'essences variées. Leur erreur est la même que celle des vieux naturalistes qui définissaient les êtres d'après leurs caractères extérieurs. Il faut maintenant écarter les branches et pénétrer dans leur ombre étouffante pour entrevoir la souche » (p. 141).

3 Rappelons que Henri Hubert est né à Paris en 1872 dans une famille de la bourgeoisie d'affaires comptant également des hommes de lettres en son sein. Brillantes études au lycée Louis-le-Grand, École normale supérieure, agrégation d'histoire en 1895. Sa passion pour les langues et les civilisations antiques le conduit à l'École pratique des hautes études où il rencontre Marcel Mauss et où il occupera à partir de 1901 une chaire d'histoire des peuples sans écritures. Son parcours savant est notamment marqué par son activité de conservateur au Musée des antiquités nationales de SaintGermain-en-Laye où il restructure les collections archéologiques, notamment la célèbre "salle de Mars" où se concentrent les données chronologiques, anthropologiques et géographiques sur le néolithique. Actif recenseur de L’Année sociologique et de la Revue archéologique, la visibilité de son œuvre souffre de l'ombre portée par celles du maître Durkheim et de l'ami Mauss avec lequel il a coécrit des textes décisifs sur la magie et le sacrifice. Le présent recueil de textes est sans doute le premier à rendre justice à cette 
œuvre prolixe qui n'a pu mener à son terme ses grands projets d'anthropologie historique des mondes celte et germain. L'utile bibliographie en fin de livre donne la mesure de l'ampleur de la tâche : rien que pour la première décennie du siècle, on compte à son actif une cinquantaine de comptes rendus par an en moyenne! Des ouvrages et articles savants, principalement en langues allemande et anglaise, qui se déploient sur un large spectre de connaissances, de l'épigraphie des cultures antiques de la Méditerranée et du Moyen-Orient au folklore nord-européen en passant par l'anthropologie des totems et tabous de par les continents. Citons seulement quelques grands noms d'auteurs recensés à plusieurs reprises pour fixer les idées: Julius Wellhausen, Herman Gunkel, James Frazer, Robertson Smith, Morris Jastrow, William Ridgeway, Salomon Reinach, Joseph Halévy, Arnold Van Gennep.

4 Outre «l'introduction" au manuel de Chantepie, le recueil contient donc d'abord l'entrée "Magia» du Dictionnaire des antiquités grecques et romaines (1904). Même tentative de définition préalable en manière de construction d'objet, même rigueur analytique dans l'exploration des formes, même prudence dans l'inférence de traits structuraux. L'enquête sur les compétences de manipulation des substances et des esprits propres à l'entreprise magicienne fouille les innombrables figures antiques de goètes, médecins, pharmaciens, sorciers et autres thaumaturges en décelant les formes qui les distinguent sur le plan de leurs ambitions et influences sociales et divines. De proche en proche se dessinent les traits qui séparent la magie de la religion par les entités mises en mouvement: individu-art-chaos-démons d'un côté, société-règleordre-dieux de l'autre.

5 Le troisième texte revient sur la représentation du temps, une étude parue dans les Mélanges d'histoire des religions publiés en 1909 avec Mauss. François-André Isambert avait également sorti de l'ombre cette éclairante étude. Une enquête à travers les calendriers, qui part de l'antinomie de principe entre temps divisible et limité des hommes et temps indivis et infini du sacré, et débouche sur les échanges entre les deux plans que les rites et les conventions calendaires opèrent (Revue française de sociologie, 20/1, 1979). Au-delà des réflexions philosophiques sur les intensités variables du temps et du sentiment de la durée, la perspective sociologique imprime sa marque précisément à travers tous les marqueurs du temps et les transferts réciproques de signes entre l'observation astrale et l'observance rythmée des rites.

6 Le quatrième et dernier texte exhumé est la longue préface à l'essai de Stefan Czarnowski sur le Culte des héros et ses conditions sociales (1919). Ce livre classique du sociologue polonais, fidèle des cours de Hubert, démontre comment se construit la figure d'un héros national, en l'occurrence Saint Patrick en Irlande. Une construction éminemment sociale dès lors que sa légende résulte de la création propre d'un corps de chantres et de poètes, les filid qui se distinguent par là même des druides en territorialisant les ordonnancements du monde. La préface insiste sur les vertus de la méthode inductive consistant à esquisser les traits de l'héroïsation à partir d'un minutieux travail de collecte centrée sur le cas irlandais. Le « Penser par cas » plus tard théorisé par Jean-Claude Passeron et Jacques Revel (2005) est ici en marche après la synthèse inaugurale de Durkheim sur Les Formes élémentaires de la vie religieuse (1912). À l'instar de son article sur la magie, Hubert témoigne dans son commentaire d'une étourdissante érudition pour approcher de ce que pourrait être une théorie anthropologique du héros comparable aux débats académiques du moment sur les liens entre totem et clan. Morts qui ne meurent jamais, à l'image des dieux, les héros 
forment ainsi l'étendard de solidarités claniques qui fait vivre un territoire national au fil de ses déploiements rituels de mémoire.

7 La signature de cet ouvrage aurait très bien pu revenir à Hubert lui-même qui occupe par ses textes les trois quarts du volume. Ce n'eût été que justice et faire honneur à la mémoire d'un auteur hier encore menacé d'oubli. Les articles sont réédités à l'état brut; ils ne comportent pas de notes diachroniques, synchroniques ou de traduction, sauf un rappel génétique d'une édition à l'autre pour l'étude sur le temps. Seules quelques notices liminaires présentent succinctement leur origine et leur réception. Mais l'ensemble se tient par l'association d'une introduction biographique générale écrite à trois (Benthien, Bert, Lorre), des annexes qui reviennent sur la position de l'auteur dans la controverse sur le totémisme et qui ouvrent aussi une petite fenêtre sur la manière dont le savant travaillait au jour le jour.

Benthien et Lorre restituent ainsi de façon très claire le débat scientifique qui a confronté Hubert à Durkheim et à Reinach, ses deux mentors, à propos du totémisme comme forme élémentaire de religion. Ils rappellent d'abord comment l'idée totémique est née dans le monde savant du second XIX ${ }^{e}$ siècle avec les thèses de Mac Lennan sur le lien univoque entre totem et clan ou de Frazer sur le totem comme manifestation de l'associationnisme primitif. Reprenant la question de la religion élémentaire dans le cadre de ses travaux sur les Grecs, les Romains, les Celtes et les Germains, Hubert se signale par la résistance à tout évolutionnisme abstrait. Les aborigènes australiens ne sont pas des proto-Grecs comme pourrait le laisser penser Reinach. De même, il n'y a pas lieu selon lui de construire une théorie sociologique de la religion, comme Durkheim tentera de le faire, à partir de l'improbable forme élémentaire que constituerait le totémisme. D'une certaine manière, le disciple est plus royaliste que le roi en rappelant sans cesse, et à rebours de toute inférence hâtive, toute particularité religieuse à sa construction sociale spécifique. Bert, de son côté, nous montre l'usage que l'historien anthropologue fait des 18000 fiches de références plus ou moins annotées qu'il accumule au fil des ans. Collecte, tri et classement de fiches dont nous apercevons quelques clichés qui font inexorablement penser aux pratiques magiques elles-mêmes dont il est précisément question. Imposante base de données redistribuée à chaque question et qui constitue aussi une sorte de garde-fou contre les généralisations hâtives. Quelques documents d'archives complètent heureusement le dossier : correspondance avec Chantepie de la Saussaye, Salomon Reinach, Henri Berr ; projets d'écriture et de cours, etc.

9 L'histoire des sciences sociales du religieux s'enrichit donc ici d'un volume précieux qui met en lumière la rigueur, la diversité et la finesse d'une œuvre trop tôt interrompue. On en saura gré à cette réédition, même si nous regrettons que le commentaire reste finalement un peu court sur les raisons pour lesquelles Hubert est resté si longtemps dans l'ombre. Nous avons par ailleurs déjà évoqué l'utile et rare bibliographie de l'auteur en fin de volume. Work in progress, nous nous permettrons seulement de signaler une référence manquante : l'entrée « Hubert Henri » du Dictionnaire des sciences religieuses sous la direction de François Laplanche (Beauchesne, 1996), notice biographique et intellectuelle rédigée par Michel Despland. 